\title{
Escrevendo uma História de Israel em X aEC: notas arqueológicas sobre a afiliação territorial de Khirbet Qeiyafa
}

\author{
Writing an History of Israel in X BC: \\ archaeological notes about the territorial affiliation of Khirbet Qeiyafa
}

Silas Klein Cardoso*

\begin{abstract}
Resumo
Um dos mais importantes achados de nosso milênio para a arqueologia das terras da Bíblia, sem dúvida, é Khirbet Qeiyafa. O sitio apresenta características da transição do Ferro I para o Ferro IIA e assente com o período das histórias bíblicas de Davi e Saul. Um dos fatores mais presentes na discussão é a filiação politico-territorial nesse momento chave da historia israelita. Fazendo fronteira entre o território filisteu, benjaminita e judaíta, o sitio teria sido extensão do território da dinastia saulida ou davidida? Apresentamos, neste ensaio, uma proposta da historia da pesquisa e dos achados.
\end{abstract}

\section{Palavras-chave}

História. Arqueologia. Khirbet Qeiyafa. Saul. Davi.

\begin{abstract}
One of the most importante findings of our millennium to the Archaeology of the Biblical Lands is Khirbet Qeiyafa. The site offers a glimpse of the transition of the Iron Age I to the Iron Age IIA and connects to the period of Saul and David. Onde of the most argued topics is about the filiation of the site in the period. In the border of the Philistine, Benjaminite and Judahite territory, the site would be a extension of a Saulid or a Davidic dynasty? We present, in this article, a proposition of the history of resource and findings.
\end{abstract}

\section{Keywords}

History. Archaeology. Khirbet Qeiyafa. Saul. David.

\section{Introdução}

A escavação do sítio de Khirbet Qeiyafa, um posto militar entre o território israelita e filisteu entre os séculos XI e X a.E.C., reavivou duas das mais perniciosas questões da interpretação bíblica contemporânea: (1) é possível acessar a realidade

[Texto recebido em setembro de 2016 e aceito em junho de 2017, com base na avaliação cega por pares realizada por pareceristas ad hoc]

* Doutorando em Ciências da Religião (UMESP). Bolsista CAPES. E-mail: silasklein@gmail.com 
histórica dos reinados de Saul e Davi através dos textos bíblicos? e (2) quais as contribuições da arqueologia para a reconstrução desse importante momento da memória israelita? Diversas teorias foram formadas a partir dos vestígios encontrados ali. O presente ensaio avalia as descobertas e interpretações das escavações de Yosef Garfinkel e Saar Ganor, realizadas entre 2007-2013, para encontrar pistas sobre a afiliação territorial da fortificação, em busca de contribuir à escrita da história de Judá/Israel entre os séculos XI e X a.E.C.

\section{Uma história das nomeações de Kh. Qeiyafa}

O sítio de Khirbet Qeiyafa se notabilizou primeiramente em 1868, nas explorações do pesquisador francês Victor Guérin, que a descreveu como um "matagal numa colina com os restos de uma vila sem importância". Em 1883, dois oficiais britânicos, Claude R. Conder e Horatio H. Kitchener, a descreveram o lugar como um "montão de pedras". ${ }^{1}$ Da identificação, apenas o nome Khirbet Kaiafa foi ressaltado por Guérin, enquanto os oficiais britânicos o chamaram "Khurbet Kiafa", cuja tradução seria "as ruínas do rastreamento de pegadas". Na arqueologia histórica do século XX d.C. o sítio foi deixado de lado por grandes pesquisadores como W. F. Albright, B. Mazar, Y. Aharoni e Z. Kallas. ${ }^{2}$ Nas décadas de 1980 e 1990, diversas explorações superficiais [surveys] foram conduzidas por Yehudi Dagan e Z. Greenhut. ${ }^{3}$ Dagan identificou, em 1992, a cidade como a bíblica Aditaim [Adithayim], mencionada em Js 15.36. A lógica de Ganor para a identificação foi a ordem geográfica das cidades, sobre a pressuposição de que os nomes estariam em ordem geográfica, como afirmavam pesquisas anteriores. ${ }^{4}$

Um novo interesse pelo sítio de Khirbet Qeiyafa surgiu em 2005, quando Saar Ganor notou estruturas da Idade do Ferro no local. Em 2008, Saar Ganor e Yosef Garfinkel começaram as escavações na localização, financiados pelo Instituto de Arqueologia da Universidade Hebraica de Jerusalém. Nessa primeira fase, foram identificados dois portões, um de quatro câmaras, junto a uma muralha casamata e dois edifícios, na parte oeste do sítio (Área B), e o outro na parte leste do sítio (Área C)..$^{5} \mathrm{O}$ achado do segundo portão foi fundamental para a identificação do sítio por Garfinkel e Ganor. Eles, que em

1 KELLEY, J. L. Khirbet Qeiyafa. In: BARRY, J. D.; BOMAR, D.; BROWN, D. R.; KLIPPENSTEIN, R.; MANGUM, D.; WOLCOTT, Carrie Sinclair; WENTZ, Lazarus; RITZEMA, Elliot; WIDDER, Wendy (Orgs.). The Lexham Bible Dictionary. Bellingham: Lexham Press, 2012, 2013, 2014, 2015 [digital edition].

2 GARFINKEL, Y.; GANOR, S. Khirbet Qeiyafa: Sha'arayim. The Journal of Hebrew Scriptures, v. 8, Art. 22, 2008, p. 2.

3 DAGAN, Y. Khirbet Qeiyafa in the Judean Shephelah: Some Considerations. Tel Aviv 36, 2009, p. 69.

4 P. ex., NA'AMAN, Nadav. The Kingdom of Judah Under Josiah. In: NA'AMAN, Nadav. Ancient Israel and its Neighbors: collected Essays. v. 1. Indiana: Eisenbrauns, 2005 [1991]. p. 329-398. No artigo, Na'aman discute as listas de cidades de Josué, defendendo que não havia subentendida uma divisão entre as cidades de Judá e Benjamim, e que o autor teria em mãos apenas uma lista de cidades, cobrindo o território de Judá no tempo de Josias, mas inscrevendo-a ao período de Josias. A divisão entre Judá e Benjamin, na lista, seria responsabilidade desse autor, que teria dividida entre dois textos: Js 15.21-62 e Js 18.21-28.

5 GARFINKEL; GANOR, 2008, p. 3. 
2007 haviam insistido na identificação do sitio como a bíblica Azeca, ${ }^{6}$ perceberam a particularidade dos dois portões e o identificaram com Saaraim [Sha ‘arayim], pelo nome significar "dois portões". Segundo os arqueólogos, Qeiyafa é a única cidade em Judá e Israel com dois portões e o nome já havia sido sugerido por David Adams (Concordia Seminary) e Anson Rainey (Tel Aviv University). ${ }^{7}$

Na'aman refutou a identificação com Saaraim, demonstrando que o sufixo "ayim" em topônimos não é um dual, mas pode ser final locativo na forma nominal. Também ressaltou que o portão encontrado poderia nem mesmo ser um portão. ${ }^{8} \mathrm{E}$, em outro artigo, propôs a identificação da cidade com a bíblica Gob. ${ }^{9}$ A proposta utiliza o mesmo arrazoado da identificação de Khirbet Qeiyafa: a citação bíblica das cidades próximas ao Vale de Elah [BJ: Vale do Terebinto] de confronto com os filisteus. Entretanto, ao invés de utilizar a narrativa de $1 \mathrm{Sm}$ 17.1-2, utiliza o texto de 2Sm 21.18-19.10 A razão para a mudança é que o texto de $1 \mathrm{Sm}$ teria sido escrito depois da consolidação do reino de Judá, quando Soco e Azeca já eram cidades judaítas, e retratariam uma realidade posterior. Entretanto, ele acentua que as duas versões do enfrentamento de Golias podem ajudar a recuperar uma história mais antiga. Como é amplamente reconhecido, a história entre Davi e Golias teriam redação tardia. Assim, a história onde Elcanã enfrenta Golias poderia representar um extrato mais antigo de memórias, talvez com locais mais antigos, ainda mais quando demonstrada a ligação entre os dois relatos. ${ }^{11}$

Em artigo mais recente, Yigal Levin propôs a identificação da cidade com o termo hebraico " ma ' $g a l$ ". Para o autor, a relação com 1 Sm 17 continua a ser evidente, entretanto, não como um topônimo do local, mas sim com uma descrição de um posto militar

6 DAGAN, 2009, p. 78.

7 Eles ressaltam que mesmo em cidades muitas vezes maiores, como Meguido e Laquis, tem apenas um portão. GARFINKEL; GANOR, 2008, p. 3.

8 NA'AMAN, Nadav. Shaaraim: The Gateway to the Kingdom of Judah. The Journal of Hebrew Scriptures, v. 8, Art. 24, 2008, p. 2-5.

9 NA'AMAN, Nadav. In Search of the Ancient Name of Khirbet Qeiyafa. The Journal of Hebrew Scriptures, v. 8, Art. 21, 2008, p. 2-8.

10 Outra razão para suspeitar da identificação com Saaraim foi levantada por Finkelstein e Fantalkin, que disseram que o séc. XI AEC seria uma data demasiadamente próxima para escrita da história. Cf. FINKELSTEIN, Israel; FANTALKIN, Alexander. Khirbet Qeiyafa: An Unsensational Archaeological and Historical Interpretation. Tel Aviv 39, 2012, p. 48. A leitura regressiva, conceito de Marc Bloch, seria um método mais apurado. Cf. FINKELSTEIN, Israel. Digging for the Truth: Archaeology and the Bible. In: SCHMIDT, Brian B. (Ed.). The Quest for the Historical Israel: Debating Archaeology and the History of Early Israel, with Israel Finkelstein \& Amihai Mazar. Leiden/Boston: Brill, 2007. p. 9-20. Uma perspectiva nacional: KAEFER, J. A. A Bíblia, a Arqueologia e a História. In: CARNEIRO, M. (Org.). Bíblia e Cultura: tradição, tradução e exegese - debatendo as diferentes leituras da Bíblia. São Paulo: Fonte/ABIB, 2014. p. 149-166.

11 Cf. Na'aman: (a) nos dois relatos o guerreiro israelita é descrito como PERSONAGEM 1 (Davi, Elcanã) filho de PERSONAGEM 2 (Jesse, Ya`are/Ya ir) o Belemita; (b) o guerreiro filisteu é introduzido por seu nome completo, Golias de Gat; (c) a arma de Golias é descrita com as mesmas exatas palavras, "a madeira de sua lança era como cilindro de tecedeira" (cf. BJ). Muitos, assim, assumem que uma real identidade de Davi seja Elcanã e o nome Davi fosse um título ou nome de reinado ou, mesmo, um local. De qualquer forma, o relato de 2Sm seria mais antigo. NA'AMAN, 2008, Art. 21, p. 3-4. 
avançado, cuja pertença étnica não seria clara. Ele demonstra que a palavra ma 'gal surge em 1Sm 17.20 e que é uma variante de ' $g l$, que significaria "circular" (1Rs 7.23) ou "bezerro" (Êx 32.4; Mq 6.6; Ml 3.20 etc). Como o termo não existe na LXX, ele mostra que a palavra foi transliterada por Magala na Vulgata. Assim, alguns intérpretes, como Keel e Bar-Efrat, assumiram a incomum característica redonda do sítio, mesmo sem ter conhecido o sítio. Tudo isso levou Levin a assumir que Khirbet Qeiyafa surge, sim, no texto de 1Sm 17.20, pela sua descrição. ${ }^{12}$

\section{Excurso: debate sobre a cronologia}

Todas essas suposições, é necessário apontar, pautam-se sobre a datação do sítio, especialmente aquelas ligadas à ocupação do Ferro I. ${ }^{13}$ Garfinkel e Ganor apresentaram os resultados de radiocarbono de quatro caroços de azeitona, cujos resultados seriam, na cronologia absoluta: 1026-975 AEC (59,6\%) ou 1051-969 AEC (77,8\%), mas como o identificaram como site do Ferro IIA, eles só mantiveram as datas depois de 1000 AEC, ficando assim com 1000-975 AEC (56,6\%) ou 1000-969 AEC (77,8\%), assinalando o sítio ao Rei Davi (1000-965 AEC). ${ }^{14}$ Israel Finkelstein e Eli Piasetzky refutaram essa interpretação dos dados, defendendo que o sítio seria do final do Ferro I, entre 1050-915 AEC. ${ }^{15}$ Leitura que condiz com a cronologia relativa da cerâmica traçada por Lily Singer-Avitz, que diz que o sítio não apresenta nenhuma cerâmica exclusiva do período do Ferro IIA. ${ }^{16}$ Garfinkel, em coautoria de Hoo-Goo Kang, reafirmou sua posição, defendendo que o debate entre "Idade do Ferro I tardia" ou "Idade do Ferro IIA muito inicial" seriam nada mais do que "diferenças conceituais de como os vestígios arqueológicos foram criados e como são divididos por pesquisadores modernos" e defende que as diferenças de Khirbet Qeiyafa marcam o início de uma nova era, o Ferro IIA. ${ }^{17}$ Mesmo que, anteriormente houvesse defendido sua metodologia, que gerou novas refutações de Finkelstein e Singer-Avitz, ${ }^{18}$ Garkinfel, juntamente a Ganor, Streit e Reimer aceitou as deficiências do procedimento

2 LEVIN, Yigal. The Identification of Khirbet Qeiyafa: A New Suggestion. BASOR 367, 2012, p. 73-86.

13 O sítio tem quatro fases ocupacionais: Bronze Médio, final do Ferro I (ou início do Ferro IIA, cf. discussão), Período Persa e Helenístico. Cf. FINKELSTEIN; FANTALKIN, 2012, p. 38-63. Dagan assinalou: BM, Ferro I-IIB, Persa, Helenístico e Garfinkel e Ganor, assinalaram: Bronze Médio; Ferro IIA, Persa e Helenístico. Cf. DAGAN, 2009, p. 69; GARFINKEL, Y; GANOR, S. Khirbet Qeiyafa in Survey and in Excavations: A Response to Y. Dagan. Tel Aviv 37, 2010, p. 67-78.

14 GARFINKEL; GANOR, 2008, p. 3.

15 FINKELSTEIN, I.; PIASETZKY, E. Khirbet Qeiyafa: Absolute Chronology. Tel Aviv 37, 2010, p. 84-88.

16 SINGER-AVITZ, Lily. The Relative Chronology of Khirbet Qeiyafa. Tel Aviv 37, 2010, p. 79-83; as escavações de Beth-Shemesh, de poucos quilômetros ao norte e de mesma época, trariam resultados similares no nível 4, cf. McCARTER; P. Kyle; BUNIMOVITZ, Shlomo; LEDERMAN, Zvi. An Archaic Ba`1 Inscription from Tel Beth-Shemesh. Tel Aviv 38, 2011, p. 35-49.

17 GARFINKEL, Yosef; KANG, Hoo-Goo. The Relative and Absolute Chronology of Khirbet Qeiyafa: Very Late Iron Age I or Very Early Iron Age IIA?. IEJ 61, 2011, p. 171-183.

18 Tréplicas em: FINKELSTEIN; FANTALKIN, 2012, p. 38-63; SINGER-AVITZ, Lily. Khirbet Qeiyafa: Late Iron Age I in Spite of it All. IEJ 62, 2012, p. 177-185. 
anterior, $^{19}$ mas reiterou sua posição em 2015, a partir da datação de Radiocarbono de dezessete caroços de azeitona dentro de um jarro. Entretanto, as duas posições sobre a data (1031-992/1064-970 AEC início e 1004-957/1010-916 AEC destruição para Garfinkel; e c. 1050-950 para Finkelstein) mantêm-nos no tempo de Davi e Saul, dadas as variáveis proposições sobre as datas destes dois reinados descritos no texto bíblico.

\section{Pertença territorial de Kh. Qeiyafa}

A identidade étnica dos habitantes de Kh. Qeiyafa, tal qual sua filiação políticoreligiosa são dois dos assuntos mais discutidos e controversos da pesquisa recente. A pesquisa despontou em duas direções opostas: de um lado os defensores da alta cronologia (Ferro IIA $=c .1000+\mathrm{AEC}^{20}$ ) e, do outro, da baixa cronologia (Ferro IIA $=c$. $925+\mathrm{AEC}^{21}$ ), todos seguros nos resultados de Carbono $14 .^{22}$ Como era de se esperar, ${ }^{23} \mathrm{o}$ primeiro grupo defendeu a pertença judaíta, enquanto o segundo grupo defendeu a pertença norte-israelita. Carecendo de uma reconstrução mais radical da história temos uma terceira via que propôs a pertença cananita e redação tardia. Um consenso é de que o site não seja filisteu, tanto pela coleção faunal ${ }^{24}$ - ausência de ossos de porco - quanto pela arquitetura. Portanto, a disputa fundamenta-se na interpretação da cultura material do sítio e compreensão dos textos bíblicos e correlatos. ${ }^{25}$ Uma dificuldade, porém, é que tais opiniões se fundamentam principalmente em métodos comparativos, como padrões

19 A falha assumida seria de que "was needed,..., was a secure context containing a large number of shortlived samples", visto que anteriormente haviam "collected from various contexts e thus should not be averaged". cf. GARFINKEL, Yosef; STREIT, Katharina; GANOR, Saar; REIMER, Paula J. King David's City at Khirbet Qeiyafa; Results of the Second Radiocarbon Dating Project. Radiocarbon 57, n. 5, 2015, p. 881-890.

20 Assumida por Garfinkel em GARFINKEL; KANG, 2011, p. 171-183.

21 Cf. discussão a partir de: FINKELSTEIN, Israel. The Archaeology of the United Monarchy: An Alternative View. Levant 28, 1996, p. 177-187; MAZAR, Amihai. Iron Age Chronology: A Reply to I. Finkelstein. Levant 29, 1997, p. 157-167; MAZAR, Amihai. The Iron Age Chronology Debate: Is the Gap Narrowing? Another Viewpoint. NEA 74, 2011, p. 105-110; FINKELSTEIN, Israel. The Iron Age Chronology Debate: Is the Gap Narrowing? NEA 74, 2011, p. 50-54.

22 Sobre a dificuldade de datação via C14, cf. SINGER-AVITZ, Lily. Carbon 14 - The Solution to Dating David and Solomon? BAR 35, v. 3, 2009, p. 28, 71.

23 Embora as alcunhas "minimalista" e "maximalista" sejam rótulos de oposição, talvez seja necessário reiterar que nenhum dos mais proeminentes autores das vertentes identificam-se sob tais categorias. Finkelstein reconhece a si próprio como centrista, enquanto Garfinkel diverge dos maximalistas, ao não assumir o relato da Monarquia Unida, embora demonstre não saber dos reais inícios Israel Norte. Nadav Na'man, que faria uma terceira via, mas de opinião, na matéria, similar a Garfinkel, também é centrista. Sobre o "centrismo" confira: FINKELSTEIN, 2007, p. 9-20. Uma perspectiva nacional: MENDONÇA, Elcio V. S. O Novo Paradigma Arqueológico e os Estudos Bíblicos. VOICES 38, v. 3-4, jul./dez. 2015, p. 25-37. Sobre a posição de Garfinkel: GARFINKEL, Yosef. The Davidic Kingdom in Light of the Finds at Khirbet Qeiyafa. City of David Studies of Ancient Jerusalem. 2011, p. 6:13-35 (English), 31-51 (Hebrew); GARFINKEL, Yosef. Khirbet Qeiyafa after five seasons of excavations. 2011, slide 3. Disponível em: <http:/ / qeiyafa.huji.ac.il/Reports/Qeiyafa2011.pdf>. Acesso em: 9 mar. 2015.

24 Embora a ausência de ossos de porcos seja uma marca distintiva aos sítios imediatos da Filistia, há ausência de ossos de porcos em sítios no coração da Filistia Cf. a discussão abaixo.

25 Especificamente a receptividade ou rejeição do conceito de Monarquia Unida. 
arquitetônicos. Assim, primeiramente elencaremos os argumentos das três principais proposições para, num segundo momento, anotarmos nossa impressão.

Nadav Na'aman e Ido Koch ${ }^{26}$ defenderam que o sítio tivesse pertença cananita. A partir do pressuposto de que houve um reflorescimento cananita que modificou as cidades-estado do Bronze Tardio, especialmente nos vales do norte, transformando-as em novos centros no Ferro I, a chamada teoria da "Nova Canaã", 27 o autor demonstra que a interação entre filisteus e cananitas, além de Israelitas/Judaítas, tem sido negligenciada, mesmo sendo ressaltada no texto bíblico (1Sn 23.1-13; 27.8-12; 13.17-18 etc). A partir desse fundamento, ele expõe seus principais argumentos: (1) o planejamento do sítio e fortificação, com muralhas casamatas seria característico de Moab e, posteriormente, de Benjamin; (2) arquitetura, com um único edifício com pilastras, que era característico de sítios das montanhas; (3) cerâmica, que não teria o collared rim-jar, típico das montanhas e, assim, se assemelharia mais à cultura material da Sefelá; (4) hábitos alimentares que não contemplavam o porco, sendo comum em populações com ancestrais pastoris e cananitas; (5) tradições literárias, que não seriam desenvolvidas na região montanhosa e todas seriam de sites cananitas; (6) semelhança com outros centros cananitas, como em Tel Kinrot. A posição tão ao sul seria pela defesa contra filisteus.

Yosef Garfinkel defende a pertença judaíta. Seus argumentos são:28 (1) o planejamento do sítio com um cinto de casas adjacentes ao muro da cidade e formando uma casamata, como em Tell en-Nasbeh, Tell Beit Mirsim e Beersheba; (2) hábitos alimentares, distintos aos filisteus, que não contemplavam porco e assadeiras encontradas nas casas com tipologia diferente dos filisteus; (3) administração, com mais de 600 alças com impressões [stamped jar handles], que seriam apenas encontradas em Judá; (4) escrita e linguagem, que teriam, p. ex., a três palavras em hebraico; (5) localização geopolítica, que faria fronteira entre Judá e Filistia, observando o Vale de Elah, principal rota da planície costeira para a região montanhosa de Jerusalém, Belém e Hebron e a 12 km de Gat; (6) culto, sobre o qual teriam encontrado dois "modelos de santuários" encontrados no templo perto ao portão principal, tendo porta proporcional à do Segundo Templo, uma decoração de três quatros escalonados e sete grupos de triglifos (vigas desde o teto com

26 NA'AMAN, Nadav. Khirbet Qeiyafa in Context. UF 42, 2010, p. 497-526; KOCH, Ido. The Geopolitical Organization of the Judean Shephelah during Iron Age I-IIA. Qathedra 143, 2012, p. 45-64. Argumentaremos especialmente sobre o artigo de Na'aman.

27 Na'aman não considera a destruição do sistema. Sobre início e destruição da "Nova Canaã", Cf. FINKELSTEIN, Israel. O reino esquecido: Arqueologia e História de Israel Norte. São Paulo: Paulus, 2015. p. $46-55$.

28 GARFINKEL, Yosef. Why Khirbet Qeiyafa is a Judean City. 2013 Disponível em: <http://qeiyafa.huji.ac.il/Reports/Is_Khirbet_Qeiyafa_a_Judean_site.pdf>. Acesso em: 9 mar. 2015; um artigo anterior com alguns argumentos similares: GARFINKEL, 2011, p. 6; Cf. também: HAGYOKOVACS, Peter. Urban Planning as Public Policy in Iron II Judah. 2012. $90 \mathrm{f}$. Thesis (The Institute of Archaeology) - Hebrew University of Jerusalem, 2012; HAGYO-KOVACS, Peter (Diretor). Khirbet Qeiyafa: A Fortified City of the Kingdom of Judah. National Geographic, 2013. Disponível em: <https://www.youtube.com/watch?v=XYsWPWZIHXc>. Aceso em: 11 mar. 2015. 
três pranchas) que explicariam 1Rs 7 e, também, não teriam sido encontradas estatuetas antropomórficas, como em Meguido, Jezrael, Hasor e Filistia.

Israel Finkelstein propôs a pertença norte-israelita. ${ }^{29}$ Seus argumentos partem, primeiramente, de refutações de três proposições dos últimos autores, para ele: (1) a cerâmica é típica da região entre Sefelá e terras altas; ${ }^{30}$ (2) os hábitos alimentares já não iluminam mais a pertença, de acordo com novos estudos zooarqueológicos; ${ }^{31}$ (3) pela escrita e linguagem é difícil de se determinar a pertença, visto não haver certeza de ser hebraico ou proto-cananita e pela tradição da escrita proto-cananita na Sefelá. ${ }^{32}$ Os três tópicos, portanto, não ajudariam na identificação. Para ele, Israel e Judá são os únicos candidatos. Embora Judá seja mais próxima, a região era distante do centro político e pouco habitada, não tendo mão de obra suficiente para a construção da fortaleza. Também, não teriam sido encontrados outros sítios fortalecidos de pertença Judaíta no período. Finkelstein associa Khirbet Qeiyafa a uma entidade territorial norte-israelita nascente de Gabaon/Gabaá. Para isso, oferece quatro argumentos: (1) a densidade habitacional da região, que teria mão de obra necessária à construção; (2) a região do platô de Gabaon-Betel, mesmo pequena, tem uma forte concentração de muralhas casamata; (3) a fortificação explicaria a presença indevida de Saul no Vale de Elah em 1Sm 17.1-3; e (4) a destruição repentina do sítio poderia ser parte da campanha do faraó Sheshonq I.

\section{Discussão}

A partir da argumentação dos autores expostas acima, podemos enxergar o seguinte panorama, sobre a identificação e pertença territorial de Khirbet Qeiyafa:

29 Argumentação principal em: FINKELSTEIN, 2015, p. 76-84; FINKELSTEIN; FANTALKIN, 2012, p. 38-63.

30 SINGER-AVITZ, 2012, p. 177-185; SINGER-AVITZ, 2010, p. 79-83.

31 Finkelstein assinala que o não consumo de porco não é mais critério distintivo, visto que pesquisas zooarqueológicas recentes mostraram que também são raros nos sítios não israelitas das planícies do Ferro I e sites rurais no centro da Filistia. Cf. FINKELSTEIN, 2015, p. 77. Em outro artigo, Hen, Bar-Oz, Gadot e Finkelstein demonstraram que a dicotomia é tardia e, o que teria sido primeiramente aplicado contra os filisteus, seria depois transferida aos norte-israelitas, que migraram quando da destruição assíria e visto o aumento do consumo de porcos em Israel no Ferro IIA. SAPIR-HEN, Lidar; BAR-OZ, Guy; GADOT, Yuval; FINKELSTEIN, Israel. Pig Husbandry in Iron Age Israel and Judah: New Insights Regarding the Origin of the Taboo. In: KAMLAH, Jens; LICHTENBERGER, Achim; WITTE, Markus. Zeitschrift des Deutschen Palästina-Vereins. 129, 2013, n. 1, p. 1-20. Sobre a distribuição geográfica de hábitos alimentares, cf. LEV-TOV, Justin S. E.; PORTER, Benjamin E.; ROUTLEDGE, Bruce E. Measuring Local Diversity in Early Iron Age Animal Economies: A View from Khirbat al-Mudayna al- Aliya (Jordan). BASOR 361, 2011, p. 67-93.

32 Cf. a discussão sobre a escrita em Qeiyafa em: BEARMAN, G.; CHRISTENS-BARRY, W. A. Spectral Imaging of Ostraca. Palarch's Journal of Archaeology of Egypt/Egyptology 6(7), 2009, p. 1-20; ROLLSTON, Christopher. The Khirbet Qeiyafa Ostracon: Methodological Musings and Caveats. Tel Aviv 38, 2011, p. 67-82; MILLARD, Alan. The Ostracon from the Days of David found in Khirbet Qeiyafa. Tyndall Bulletin 62.1, 2011, p. 1-13. Sobre a tradição contínua de administração cananita na Sefelá, cf. FINKELSTEIN, Israel; SASS, Benjamin; SINGER-AVITZ, Lily. Writing in Iron IIA Philistine in the Light of the Tel Zayit/Zeta Abecedary. ZDPV 124, v. 1, 2008, p. 1-14. 
Tabela 1: Argumentos dos principais autores sobre a pertença territorial de Khirbet Qeiyafa

\begin{tabular}{|c|c|c|c|}
\hline & Na'aman (Nova Canaã) & Garfinkel (Judá/Davi) & Finkelstein (Benjamin/Saul) \\
\hline Identificação & Gob, 2Sm 21 & Saaraim, Js 15.36 & Gob, mas impossível confirmar \\
\hline $\begin{array}{l}\text { Planejamento e } \\
\text { fortificações: } \\
\text { casamata ligadas a } \\
\text { salas amplas }\end{array}$ & $\begin{array}{l}\text { Car. Moab (el-Lehun, } \\
\text { Kh. Mudeyine } \\
\text { Muarrajeh, Kh. } \\
\text { Mudeyine Aliya, Kh. el- } \\
\text { Muammariyya) e } \\
\text { Benjamin (ed-Dawwara, } \\
\text { et-Tell, en-Nasbeh) }\end{array}$ & $\begin{array}{l}\text { Car. Judá (en-Nasbeh, } \\
\text { Beit Mirsim, Bersheba) }\end{array}$ & $\begin{array}{l}\text { Car. Moab (el-Lehun, Kh. } \\
\text { Mudeyine Muarrajeh, Kh. } \\
\text { Mudeyine Aliya, Kh. el- } \\
\text { Muammariyya), Amon (el- } \\
\text { Umeiri), terras altas ao norte de } \\
\text { Jerusalém (ed-Dawwara'en- } \\
\text { Nasbeh), Negev (Refed, Hatira) }\end{array}$ \\
\hline Alimentação: s/porco & Não filisteu & Não filisteu & Também na Filistia: inválido \\
\hline $\begin{array}{l}\text { Cerâmica: sem coll.. } \\
r-j, \text { com stamped j. } h .\end{array}$ & $\begin{array}{l}\text { Sem Collared Rim-Jar, } \\
\text { característico da Sefelá }\end{array}$ & $\begin{array}{l}\text { Com stamped jar } \\
\text { handles, Judá posterior }\end{array}$ & Car. Sefelá: inválido \\
\hline Escrita: Óstraco & Proto-cananeu, Sefelá & Hebraico, de Judá. & Sem consenso: inválido \\
\hline $\begin{array}{l}\text { Função e localização } \\
\text { geopolítica: } \\
\text { Vale de Elah }\end{array}$ & $\begin{array}{l}\text { Defesa dos cananeus } \\
\text { (Tel Kinrot) contra os } \\
\text { filisteus. }\end{array}$ & $\begin{array}{l}\text { Defesa judaíta contra } \\
\text { filisteus, proteção } \\
\text { Jerusalém/Hebron/Belém }\end{array}$ & $\begin{array}{l}\text { Defesa saulida (platô Gabaon- } \\
\text { Betel) contra os filisteus }\end{array}$ \\
\hline $\begin{array}{l}\text { Culto: sem estátuas + } \\
\text { modelos de santuário }\end{array}$ & & $\begin{array}{l}\text { Culto anicônico } \\
\text { monoteísta de Judá }\end{array}$ & \\
\hline Densidade pol. & - & Judá desenvolvida & Benjamin desenvolvida \\
\hline Destruição & Filisteus (Gat, X AEC) & - & Campanha faraó Sheshonq I \\
\hline
\end{tabular}

Alguns pontos da comparação não contribuem à identificação. Primeiramente, o debate sobre a coleção faunal do sítio (ossos de animais) é improdutiva, visto que recentemente foram descobertos outros sítios com a mesma característica fora dos conjuntos das montanhas e no centro da Filistia. Da mesma forma, a cerâmica, ao mesmo tempo há consenso que se trata prioritariamente de uma coleção de "jarras com aro de colarinho" [collared rim-jars] da Sefelá e não filistia, podem, com as "alças de jarros estampados" [stamped jar handles] remeter a uma tradição cerâmica mais antiga, ${ }^{33}$ que é reconhecida da Judá dos séculos IX-VII a.E.C. Na verdade, o layout pode ter surgido na Sefelá Superior, assim, não responde à afiliação.

Sobre o planejamento das muralhas e fortificações, a tradição arquitetônica é claramente não filisteia, mas foi interpretada de formas distintas. $\mathrm{Na}^{\prime}$ aman e Finkelstein interpretaram-na como cananita ${ }^{34} \mathrm{e}$ benjaminita, enquanto Garfinkel defendeu a pertença

33 Goren, Finkelstein e Na'aman argumentam que o as jarras $l m l k$ parecem ter tido origem na parte superior da Sefelá e, talvez, estejam ligadas à área em sua origem. Os selos de roseta (Rosette Stamped Jar), por sua vez, GOREN, Yuren; FINKELSTEIN, Israel; NA'AMAN, Nadav. Inscribed in Clay: Provenance Study of the Amarna Letters and other Ancient Near Eastern Texts. Tel Aviv: Tel Aviv University, 2004. p. $284-285$.

34 Avraham Faust se opõe à posição cananita marcada por Koch e Na'aman. Para ele, é impossível conceber que uma cultura em vias de assimilação - no caso, canaanita - fosse capaz de construir um forte das dimensões de Kh. Qeiyafa e, também, para o autor, as comparações traçadas são de um período muito 
judaíta. A análise de Garfinkel, porém, contempla apenas quatro sítios judaítas e um benjaminita (sem contar Kh. Qeiyafa), tornando seus resultados tendenciosos..$^{35}$ Entretanto, se utilizarmos tais resultados, associando-os à teoria de uma unidade políticoterritorial norte-israelita nascente no platô de Gabaon-Betel, ${ }^{36}$ teríamos que puxar Tell enNasbeh e, talvez, Beth-Shemesh ${ }^{37}$ para essa unidade. Assim, teríamos Tell en-Nasbeh, Beth Shemesh, Khirbet ed-Dawwara (unanimamente benjaminita), como sítios da unidade Gabaon-Betel, enquanto Tel Beit Mirsim e Tel Beersheba seriam judaítas. Entretanto, os últimos dois sítios foram escavados com métodos e pressupostos ultrapassados: Beit Mirsim foi escavada por Albright nos anos 1920 e a escavação de Beersheba por Aharoni datou o sítio pelo sensacionalismo de sua época. ${ }^{38}$ Assim, teríamos quatro cidades de pertença norte-israelita e duas de datação duvidosa, talvez posteriores.

Israel Finkelstein tinha certa razão ao levantar a questão da escrita. Não havia, na data de escrita de seu artigo e livro, 2012 e 2013, respectivamente, consenso sobre o idioma das inscrições do óstraco. Entretanto, desde então, outra cerâmica foi investigada e divulgada, sendo traduzida de acordo com a tradução do óstraco anterior. O conteúdo da escrita, entretanto, parece coadunar mais à teoria de Finkelstein do que à dos escavadores. Ela traz a inscrição: "'Isba 'al, filho de Beda '" ${ }^{39}$ Embora os autores do artigo não assumam, pelo nome "Beda", a associação com o bíblico 'Isba 'al, a relação é tentadora, ainda mais se observarmos o texto de $2 \mathrm{Sm}$ 2.9, que diz que Isboset ('Isba' 'al), filho de Saul, foi feito rei "para Galaad, e para os assuritas, e para Jezrael, e sobre Efraim, e sobre Benjamin, e sobre todo o Israel".

anterior, enfraquecendo o argumento. O autor, entretanto, não defende uma posição, apenas concorda que o sítio seria marca da colonização da Sefelá pelos povos das montanhas (Israel ou Judá). FAUST, Avraham. The Shephelah in the Iron Age: A New Look On the Settlement of Judah. PEQ 145, v. 3, 2013, p. 203-219.

35 A pesquisa do orientando de mestrado de Yosef Garfinkel, Peter Hagyo-Kovacs, parece ter sido protagonista na argumentação que identificou o sítio. Cf. HAGYO-KOVACS, 2012.

36 Cf. FINKELSTEIN, 2015 p. 57-84.

37 Cuja pertença político-territorial, na tradição faunal, cerâmica e arquitetônica também é difícil de se precisar.

38 Na questão do portal de seis câmaras, que já foi confirmado ser improcedente. Leia sobre a troca de datações de Aharoni sobre o Stratum V em: MANOR, D. W. Beer-Sheba (Place). In: FREEDMAN, D. N. (Org.), The Anchor Yale Bible Dictionary. New York: Doubleday, 1992. v. 1. p. 643.

39 GARFINKEL, Yosef; GOLUB, Mitka R.; MISGAV, Haggai; GANOR, Saar. The 'Isba 'al Inscription from Khirbet Qeiyafa. BASOR 373, 2015, p. 217-233. 


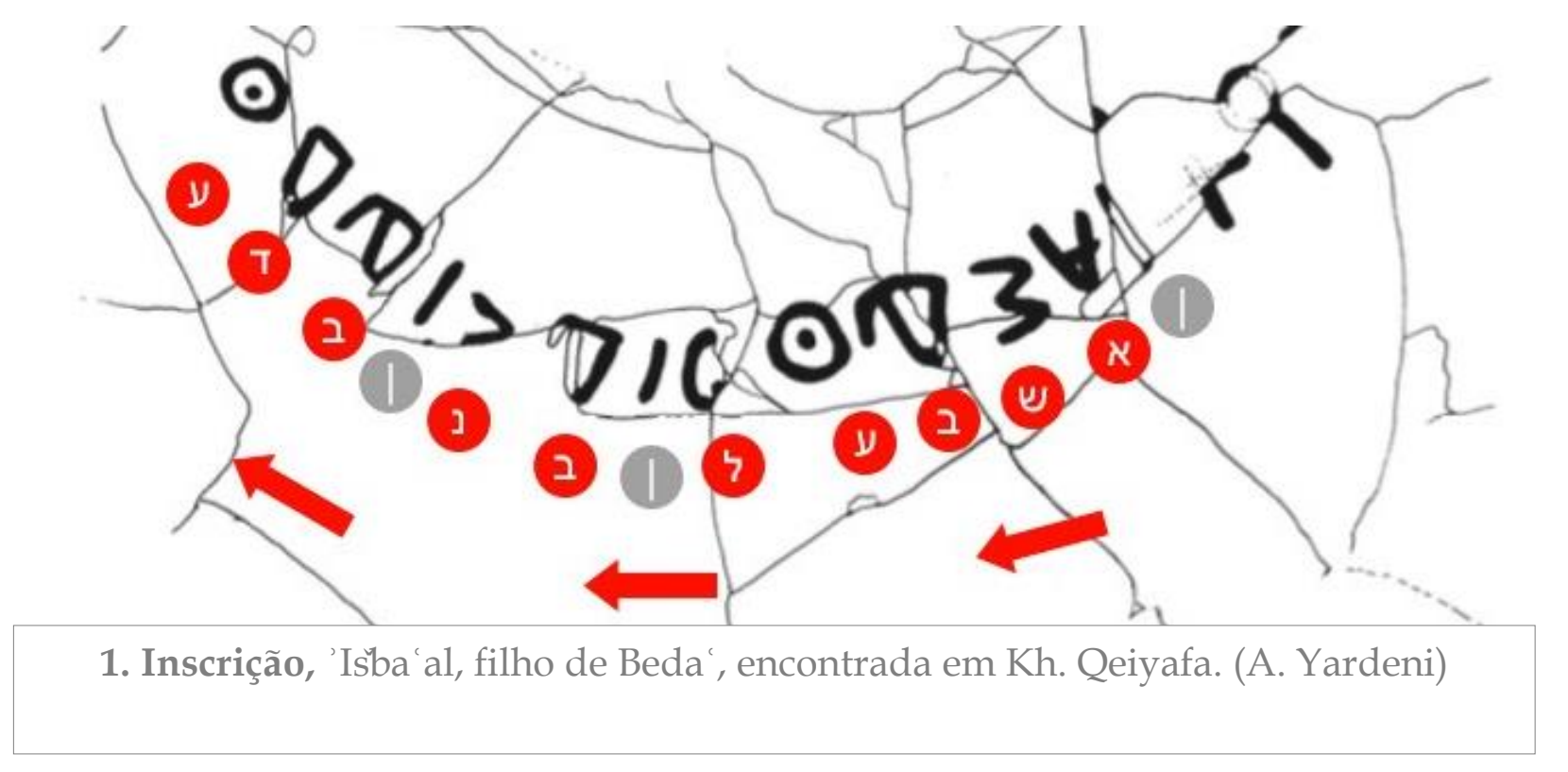

Queremos, no entanto, propor nova associação da inscrição. O texto de 1 Sm 12.11 do TM traz notável ressonância: o nome bĕdān. O nome, entre a lista de juízes de Israel Norte, foi desde a antiguidade considerado crux interpretum, i.e., de interpretação impossível, sendo "corrigido" para Baraque (gr. Barak) na LXX, para "Débora e Baraque" na Peshitta, enquanto o Talmud diz: "Bedan é Sansão". ${ }^{40}$ O que se sabe, no entanto, é que o texto pode lidar com uma antiga versão do texto bíblico não-relacionada ao formato hebraico-canônico, que une Juízes e 1 Samuel (i.e., os "profetas anteriores" ou a $O H D$ ). ${ }^{41}$ Embora a grafia seja distinta, terminando com a letra "ayin" ao invés da letra "nun" (fig. 1), o texto bíblico explicita as relações do personagem bĕdān com a região de Manassés, na Transjordânia (Cf. 1Cr 7.17, outra única ocorrência do nome), que estava sob o domínio de 'Isba'al, segundo $2 \mathrm{Sm} 2.9$, texto que coaduna com outra evidência textual do sítio, a inscrição do óstraco, cuja tradução traz os deveres do rei com os necessitados. De igual modo o som da gutural "ayin" não é plenamente compreendido, sendo um som gutural que existiria também no arábico e que seria comparado ao "som gutural de um camelo enquanto está sendo carregado". ${ }^{42}$ A coincidência de nome, região, filiação e do texto bíblico junto ao óstraco talvez revelem uma convergência histórica que ilumine o texto bíblico.

O culto de Kh. Qeiyafa só foi estudado por Garfinkel. O arqueólogo defende um culto público e um privado, mas mantém-se na opinião de que o sítio revela o culto anicônico dos tempos de Davi e Salomão, por não terem encontrados imagens femininas. ${ }^{43}$ Entretanto, a própria inscrição "'Işba 'al, filho de Beda ', já subentende a adoração ao Deus

40 FROLOV, Serge. Bedan: A Riddle in Context. JBL 126, n. 1, 2007, p. 164-167.

41 Cf. BRETTLER, Marc. The Book of Judges: Literature as Politics. JBL 108, n. 3, 1989, p. 403.

42 JOÜON, P.; MURAOKA, T. A grammar of biblical Hebrew. Roma: Pontificio Istituto Biblico, 2006. p. 25-26.

43 GARFINKEL, Y.; GANOR, S. Cult in Khirbet Qeiyafa from the Iron Age IIA - Cult Rooms and Shrine Models. New Studies in the Archaeology of Jerusalem and its Region 6, 2012, p. 50-65 (Hebrew). 
Baal. Fora isso, foram encontrados dois naoi, modelos de santuário, um refletindo as características materiais da Idade do Bronze Tardio e outro com as características, segundo Garfinkel, do Ferro I, que explicariam os triglifos do templo salomônico. ${ }^{44}$ É difícil, no entanto, assumir o aniconismo ${ }^{45}$ do sítio. Primeiro, com relação aos modelos de templo, é preciso dizer que muitos traziam estátuas de deidades ${ }^{46} \mathrm{e}$, naqueles encontrados vazios, é difícil precisar sua função. Alguns parecem representar o "aniconismo de espaço vazio", com tronos vazios, enquanto outros parecem ter sido recipientes para ofertas votivas. Eles podem, também, ter sido roubados, quando da destruição, costume comum e descrito no Prisma de Nimrod. ${ }^{47}$ De qualquer jeito, é irresponsável se pautar pela prova negativa. $\mathrm{O}$ culto, portanto, não ajuda na resposta sobre a afiliação, com os dados e pesquisas disponíveis até o momento.

\section{Conclusão}

Traçar uma afiliação territorial para Khirbet Qeiyafa a partir da arqueologia é difícil. Deve-se interpretar um rico mosaico de evidências não apenas do sítio, mas das culturas ao redor e ter acesso a uma vasta literatura especializada. Entretanto, acreditamos ser possível assinalar a pertença norte israelita. Primeiramente, é difícil recompor o nome da cidade, tal qual o senso de pertença dos habitantes do sítio. A maior probabilidade é de que a cidade seja a bíblica Gob (2Sm 21.18-19), no formato circular (heb. ma 'gal) e que tenha sido posto militar avançado de um povo das terras altas frente aos filisteu. Embora Judá esteja mais próxima geograficamente, se levarmos em conta as descrições do sítio e situação geopolítica a partir do relato bíblico (1Sm 17.20; 2Sm 21.18-19; 2Sm 2.9) e observarmos a formação arquitetônica do sítio (fortificação de casamata), podemos associá-la à entidade político-territorial de Gabaon/Gabaá, sob a dinastia saulida, pela cronologia do sítio, que encaixa-se perfeitamente à condição geopolítica da campanha de Sheshonq I no período. Os achados textuais talvez cooperem à argumentação, ainda mais se a hipótese do nome encontrado ser uma versão do nome Bedan. Em relação ao culto, é impossível afirmar, no estado atual da pesquisa e dos achados, quais eram as práticas tanto de Judá quanto de Israel Norte na época, embora a adoração a algum baal seja presumida, pelo elemento teofórico "Baal" no nome 'Isba 'al.

44 GARFINKEL, Y.; MUMCUOGLU, M. Triglyphs and Recessed Doorframes on a Building Model from Khirbet Qeiyafa: New Light on Two Technical Terms in the Biblical Descriptions of Solomon's Palace and Temple. IEJ 63, 2013, p. 135-163.

45 Veja uma inigualável monografia sobre aniconismo em: METTINGER, Tryggve. No Graven Image? Israelite Aniconism in Its Ancient Near Eastern Context. CB 42. Stockholm: Almqvist \& Wiksell International, 1997.

46 ZEVIT, Ziony. The Religions of Ancient Israel: A Synthesis of Parallactic Approaches. New York: Continuum, 2001. p. 328-343.

47 Que lê: "[Os habitantes de Samaria, que concordaram [e maquinaram] com o rei [hostil a] mim, que não fizeram o serviço e não trouxeram o tributo [a Aššur] e que não batalharam, eu lutei contra eles com o poder dos grandes deuses, meus senhores. Contei como espólio 27.280 pessoas, junto a suas carruagens, e deuses, em quem eles confiaram...". Tradução do inglês de HALLO, W. W.; YOUNGER, K. L. Context of Scripture: v. 2 Monumental Inscriptions of the Biblical World. Leiden/Boston: Brill, 2000. p. 295. 


\section{Referências}

BEARMAN, G.; CHRISTENS-BARRY, W. A. Spectral Imaging of Ostraca. Palarch's Journal of Archaeology of Egypt/Egyptology 6(7), 2009.

BRETTLER, Marc. The Book of Judges: Literature as Politics. JBL 108, n. 3, 1989.

DAGAN, Y. Khirbet Qeiyafa in the Judean Shephelah: Some Considerations. Tel Aviv 36, 2009.

FAUST, Avraham. The Shephelah in the Iron Age: A New Look On the Settlement of Judah. PEQ 145, v. 3, 2013.

FINKELSTEIN, I.; PIASETZKY, E. Khirbet Qeiyafa: Absolute Chronology. Tel Aviv 37, 2010.

FINKELSTEIN, Israel. Digging for the Truth: Archaeology and the Bible. In: SCHMIDT, Brian B. (Ed.). The Quest for the Historical Israel: Debating Archaeology and the History of Early Israel, with Israel Finkelstein \& Amihai Mazar. Leiden/Boston: Brill, 2007.

O reino esquecido: Arqueologia e História de Israel Norte. São Paulo: Paulus, 2015.

. The Archaeology of the United Monarchy: An Alternative View. Levant 28, 1996.

. The Iron Age Chronology Debate: Is the Gap Narrowing? NEA 74, 2011.

FINKELSTEIN, Israel; FANTALKIN, Alexander. Khirbet Qeiyafa: An Unsensational Archaeological and Historical Interpretation. Tel Aviv 39, 2012.

FINKELSTEIN, Israel; SASS, Benjamin; SINGER-AVITZ, Lily. Writing in Iron IIA Philistine in the Light of the Tel Zayit/Zeta Abecedary. ZDPV 124, v. 1, 2008.

FROLOV, Serge. Bedan: A Riddle in Context. JBL 126, n. 1, 2007.

GARFINKEL, Y.; GANOR, S. Cult in Khirbet Qeiyafa from the Iron Age IIA - Cult Rooms and Shrine Models. New Studies in the Archaeology of Jerusalem and its Region 6, 2012.

GARFINKEL, Y.; GANOR, S. Khirbet Qeiyafa: Sha'arayim. The Journal of Hebrew Scriptures, v. 8, Art. 22, 2008.

GARFINKEL, Y.; MUMCUOGLU, M. Triglyphs and Recessed Doorframes on a Building Model from Khirbet Qeiyafa: New Light on Two Technical Terms in the Biblical Descriptions of Solomon's Palace and Temple. IEJ 63, 2013.

GARFINKEL, Y; GANOR, S. Khirbet Qeiyafa in Survey and in Excavations: A Response to Y. Dagan. Tel Aviv 37, 2010.

GARFINKEL, Yosef. Khirbet Qeiyafa after five seasons of excavations. 2011, slide 3.

Disponível em: <http:/ /qeiyafa.huji.ac.il/Reports/Qeiyafa2011.pdf>. Acesso em: 9 mar. 2015.

The Davidic Kingdom in Light of the Finds at Khirbet Qeiyafa. City of David Studies of Ancient Jerusalem. 2011. 
Why Khirbet Qeiyafa is a Judean City. 2013 Disponível em:

<http:/ /qeiyafa.huji.ac.il/Reports/Is_Khirbet_Qeiyafa_a_Judean_site.pdf>. Acesso em: 9 mar. 2015.

GARFINKEL, Yosef; GOLUB, Mitka R.; MISGAV, Haggai; GANOR, Saar. The 'Isba 'al Inscription from Khirbet Qeiyafa. BASOR 373, 2015.

GARFINKEL, Yosef; KANG, Hoo-Goo. The Relative and Absolute Chronology of Khirbet Qeiyafa: Very Late Iron Age I or Very Early Iron Age IIA?. IEJ 61, 2011.

GARFINKEL, Yosef; STREIT, Katharina; GANOR, Saar; REIMER, Paula J. King David's City at Khirbet Qeiyafa; Results of the Second Radiocarbon Dating Project. Radiocarbon 57, n. 5, 2015.

GOREN, Yuren; FINKELSTEIN, Israel; NA'AMAN, Nadav. Inscribed in Clay: Provenance Study of the Amarna Letters and other Ancient Near Eastern Texts. Tel Aviv: Tel Aviv University, 2004.

HAGYO-KOVACS, Peter (Diretor). Khirbet Qeiyafa: A Fortified City of the Kingdom of Judah. National Geographic, 2013. Disponível em:

<https://www.youtube.com/watch?v=XYsWPWZIHXc>. Aceso em: 11 mar. 2015.

. Urban Planning as Public Policy in Iron II Judah. 2012. 90 f. Thesis (The Institute of

Archaeology) - Hebrew University of Jerusalem, 2012.

HALLO, W. W.; YOUNGER, K. L. Context of Scripture: v. 2 Monumental Inscriptions of the Biblical World. Leiden/Boston: Brill, 2000.

JOÜON, P.; MURAOKA, T. A grammar of biblical Hebrew. Roma: Pontificio Istituto Biblico, 2006.

KAEFER, J. A. A Bíblia, a Arqueologia e a História. In: CARNEIRO, M. (Org.). Bíblia e Cultura: tradição, tradução e exegese - debatendo as diferentes leituras da Bíblia. São Paulo: Fonte/ ABIB, 2014.

KELLEY, J. L. Khirbet Qeiyafa. In: BARRY, J. D.; BOMAR, D.; BROWN, D. R.; KLIPPENSTEIN, R.; MANGUM, D.; WOLCOTT, Carrie Sinclair; WENTZ, Lazarus; RITZEMA, Elliot; WIDDER, Wendy (Orgs.). The Lexham Bible Dictionary. Bellingham: Lexham Press, 2012, 2013, 2014, 2015 [digital edition].

$\mathrm{KOCH}$, Ido. The Geopolitical Organization of the Judean Shephelah during Iron Age I-IIA. Qathedra 143, 2012.

LEVIN, Yigal. The Identification of Khirbet Qeiyafa: A New Suggestion. BASOR 367, 2012.

LEV-TOV, Justin S. E.; PORTER, Benjamin E.; ROUTLEDGE, Bruce E. Measuring Local Diversity in Early Iron Age Animal Economies: A View from Khirbat al-Mudayna al- 'Aliya (Jordan). BASOR 361, 2011.

MANOR, D. W. Beer-Sheba (Place). In: FREEDMAN, D. N. (Org.), The Anchor Yale Bible Dictionary. New York: Doubleday, 1992. v. 1.

MAZAR, Amihai. Iron Age Chronology: A Reply to I. Finkelstein. Levant 29, 1997. 
2011.

The Iron Age Chronology Debate: Is the Gap Narrowing? Another Viewpoint. NEA 74,

McCARTER; P. Kyle; BUNIMOVITZ, Shlomo; LEDERMAN, Zvi. An Archaic Ba`l Inscription from Tel Beth-Shemesh. Tel Aviv 38, 2011.

MENDONÇA, Elcio V. S. O Novo Paradigma Arqueológico e os Estudos Bíblicos. VOICES 38, v. 3-4, jul./dez. 2015.

METTINGER, Tryggve. No Graven Image? Israelite Aniconism in Its Ancient Near Eastern Context. CB 42. Stockholm: Almqvist \& Wiksell International, 1997.

MILLARD, Alan. The Ostracon from the Days of David found in Khirbet Qeiyafa. Tyndall Bulletin 62.1, 2011.

NA'AMAN, Nadav. In Search of the Ancient Name of Khirbet Qeiyafa. The Journal of Hebrew Scriptures, v. 8, Art. 21, 2008.

. Khirbet Qeiyafa in Context. UF 42, 2010.

Shaaraim: The Gateway to the Kingdom of Judah. The Journal of Hebrew Scriptures, v. 8, Art. 24, 2008.

The Kingdom of Judah Under Josiah. In: NA'AMAN, Nadav. Ancient Israel and its Neighbors: collected Essays. v. 1. Indiana: Eisenbrauns, 2005 [1991].

ROLLSTON, Christopher. The Khirbet Qeiyafa Ostracon: Methodological Musings and Caveats. Tel Aviv 38, 2011.

SAPIR-HEN, Lidar; BAR-OZ, Guy; GADOT, Yuval; FINKELSTEIN, Israel. Pig Husbandry in Iron Age Israel and Judah: New Insights Regarding the Origin of the Taboo. In: KAMLAH, Jens; LICHTENBERGER, Achim; WITTE, Markus. Zeitschrift des Deutschen Palästina-Vereins. 129, 2013, n. 1.

SINGER-AVITZ, Lily. Carbon 14 - The Solution to Dating David and Solomon? BAR 35, v. 3, 2009.

. Khirbet Qeiyafa: Late Iron Age I in Spite of it All. IEJ 62, 2012.

The Relative Chronology of Khirbet Qeiyafa. Tel Aviv 37, 2010.

ZEVIT, Ziony. The Religions of Ancient Israel: A Synthesis of Parallactic Approaches. New York: Continuum, 2001. 